\title{
Correlação entre sinais e sintomas de incontinência urinária e autoestima em idosas
}

Correlation between signs and symptoms of urinary incontinence and self-esteem in elderly

women

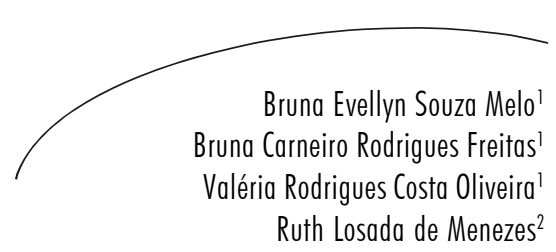

Resumo

Objetivo: Identificar a prevalência de sinais e sintomas de incontinência urinária e sua relação com a autoestima de idosas. Método: A amostra foi constituída por 27 idosas, com média de idade de 67,59 ( \pm 5,16), participantes do Curso Universidade Aberta à Terceira Idade (UNATI) do Programa Gerontologia Social da Pontifícia Universidade Católica de Goiás. Foram utilizados três instrumentos para avaliação, sendo eles o Miniexame do estado mental, um instrumento específico elaborado para este estudo e o questionário de autoestima desenvolvido por Steglich. O tratamento estatístico foi descritivo e com testes não-paramétricos, com nível de significância de $\mathrm{p}<0,05$. Resultados: Foi observado que $44,4 \%$ das idosas apresentam perda de urina, destas, $58,33 \%$ perdem uma vez por semana, todas relatam perder pouca quantidade de urina e $66,7 \%$ apresentam perda há mais de um ano. A autoestima foi considerada baixa em $77,8 \%$ das idosas, mas não houve correlação entre a mesma e os sintomas de IU. Conclusão: A autoestima diminuída é uma condição frequente na população idosa e vários fatores podem contribuir para isso, porém na amostra estudada não foi possível identificar a contribuição da IU.

\section{Abstract}

Objective: Identify the prevalence of signs and symptoms of urinary incontinence and their relationship with the self-esteem of elderly. Methods: The sample was constitute by 27 elderly, mean age $67.59( \pm 5.16)$, participants of Open University for the Elderly (UNATI) of the Social Gerontology Program of Pontifical Catholic University of Goiás. Three instruments were used for: the Mini mental state examination, a specific instrument prepared for this study and a self-esteem and self-image questionnaire developed by Steglich. The statistical treatment was

\footnotetext{
Curso de Fisioterapia, Departamento de Enfermagem, Nutrição e Fisioterapia. Pontifícia Universidade Católica de Goiás. Goiania, GO, Brasil.

2 Faculdade de Ceilândia FCE. Universidade de Brasília. Brasilia, DF, Brasil

Palavras-chave: Saúde do idoso. Saúde Mental.

Motivação. Incontinência urinária. Idosas.

Autoestima. 
descriptive and non-parametrical test, with significance level of $\mathrm{p}<0.05$. Results: It was observed that $44.4 \%$ of the elderly have urine loss, $58.33 \%$ lose once a week, all reported losing a little amount of urine and $66.7 \%$ have urine loss for over a year. The self-esteem was considered low in $77.8 \%$ of elderly, but no correlation between self-esteem and symptoms of UI was found. Conclusion: The decreased self-esteem is a frequent condition in the elderly people and several factors can contribute for this, but this sample did not allow to identify the contribution of the UI.
Key words: Health of the elderly. Mental health. Motivation. Urinary incontinence. Elderly. Self-esteem.

\section{INTRODUÇÃO}

A incontinência urinária (IU), segundo o Comitê de Padronização da Sociedade Internacional de Continência, é definida como qualquer perda involuntária de urina. É classificada em três tipos principais: a incontinência urinária de esforço (IUE), quando ocorre perda de urina durante algum esforço que aumente a pressão intra-abdominal, como tosse, espirro ou exercícios físicos; a urge-incontinência (IUU), caracterizada pela perda de urina acompanhada por forte sensação de urgência para urinar; e a incontinência urinária mista (IUM), quando há queixa de perda associada à urgência e também aos esforços. ${ }^{14}$

É altamente prevalente em adultos e de duas a quatro vezes mais comum em mulheres do que em homens. É uma alteração não inerente ao processo de envelhecimento, mas sua incidência aumenta linearmente com a idade, podendo ser considerada como uma síndrome geriátrica, devido à alta prevalência nesses indivíduos e ao impacto negativo que provoca no idoso. $3,5,6$

A idade avançada está relacionada com alterações de motivação, destreza manual, mobilidade, lucidez e existência de doenças associadas, que são fatores que podem ser responsáveis pela incontinência urinária; nas mulheres, pode ainda estar associado a mudanças hormonais que ocorrem na menopausa, aumento do índice de massa corpórea, múltiplas gestações, constipação intestinal, efeitos colaterais de medicamentos entre outros. ${ }^{3,4,7}$

Os sinais e sintomas decorrentes da incontinência urinária são: aumento da frequência miccional, noctúria, urgência, hiper-reflexia vesical, perda de urina ao esforço, incontinência no intercurso sexual, infecções urinárias e dor na bexiga. ${ }^{9}$

A incontinência urinária não está relacionada somente a comprometimentos físicos; também pode gerar consequências que atingem uma ampla esfera envolvendo aspectos psicossociais, deteriorando significativamente a qualidade de vida, limitando sua autonomia e reduzindo sua autoestima. Outros fatores como gênero, idade, condição socioeconômica e quantidade de perda de urina podem ser responsáveis por diferenças no comportamento pessoal frente à incontinência. O impacto dos sintomas está relacionado à percepção individual de cada mulher frente a severidade, tipo e quantidade da perda de urina. ${ }^{39,10}$

Problemas de ordem social, ocupacional, doméstica e sexual podem ser encontrados em mulheres com IU e são causas significativas de morbidade, estresse e debilidade, tendo grande impacto na qualidade de vida. Fazem parte do quadro clínico alterações como vergonha, depressão, isolamento, ansiedade, estresse emocional, insatisfação sexual, constrangimento social, baixo desempenho profissional e perda da autoestima. Os efeitos psicossociais podem ser mais devastadores que as consequências sobre a saúde física, podendo afetar atividades diárias, a interação social e a autopercepção do estado de saúde. $3,8,10-12$

A autoestima é a avaliação que o indivíduo faz e mantém de si mesmo, sendo construída através de interações e avaliações sociais. Alguns 
autores a definem como o senso individual de autoaceitação, capacidade e valor pessoal, sendo o julgamento sobre o próprio valor e atitude de aprovação ou desaprovação do eu. Pode ser considerada ainda como um apanhado de sentimentos e crenças do indivíduo acerca da competência e valor, de suas habilidades de tornar as coisas diferentes, de confrontar e lidar com os desafios, de sua capacidade de aprender com o fracasso e o sucesso, e evidenciar um tratamento respeitoso para com ele e os outros. ${ }^{13}$

Essas alterações de ordem psicossociais fazem com que as pacientes portadoras de incontinência urinária convivam com o problema por muitos anos sem falar sobre o assunto com familiares, amigos e profissionais de saúde. Muitas vezes, consideram uma condição natural da idade e decorrentes de gestações, além da falta de conhecimento sobre a existência de tratamento viável. Isso faz com que essas mulheres deixem de procurar serviços de saúde, sendo que a procura por atendimento está relacionada à severidade dos sintomas. ${ }^{6,7,11}$

Diante do crescente aumento da incidência de síndromes geriátricas, com destaque para a incontinência urinária, e das repercussões da mesma na vida da mulher, é necessária maior investigação sobre como as mulheres percebem a presença e suas repercussões em suas vidas. Desta forma, este estudo permitirá aos profissionais da saúde conhecer essas alterações que muito influenciam na qualidade e adesão do tratamento, favorecendo uma melhor condução do mesmo.

O objetivo deste estudo consiste em identificar a prevalência de sinais e sintomas de IU e sua relação com a autoestima de idosas.

\section{MÉTODOS}

Trata-se de um estudo analítico transversal, realizado com idosas participantes do Curso Universidade Aberta à Terceira Idade (UNATI) do Programa de Gerontologia Social da Pontifícia Universidade Católica de Goiás. A amostra por conveniência foi de 27 idosas, sendo incluídos indivíduos com idade igual ou superior a 60 anos que aceitaram participar da pesquisa, assinaram o termo de consentimento livre e esclarecido e que não possuíam déficit cognitivo que os impedisse de responder aos instrumentos, avaliado pelo Mini-Exame do Estado Mental.

Participavam do curso UNATI, no momento da investigação, aproximadamente 90 alunas idosas. No entanto, o aceite em participar da pesquisa foi acusado por um número inferior, $\mathrm{o}$ que justifica a amostra reduzida, pelo fato de essa temática muitas vezes despertar o sentimento de negação e constrangimento social por parte das idosas. Assim, respeitando-se os preceitos éticos na pesquisa com seres humanos, aquelas que não manifestaram seu aceite mediante a assinatura do termo de consentimento livre e esclarecido foram liberadas sem qualquer prejuízo.

O projeto foi aprovado pelo Comitê de Ética da Pontifícia Universidade Católica de Goiás, (00790168000-09), e a coleta de dados realizada no período de 14 de setembro a $1^{\circ}$ de outubro de 2009. Foram aplicados três instrumentos de avaliação, na seguinte ordem: Mini-exame do estado mental (MEEM), um instrumento elaborado especificamente para o estudo e o questionário de autoestima.

O MEEM é composto por questões agrupadas em sete categorias, cada uma planejada com o objetivo de se avaliarem funções cognitivas específicas. São elas: orientação para tempo, orientação para local, registro de três palavras, atenção e cálculo, evocação das três palavras registradas, linguagem e praxia vísuo-construtiva.

A cada resposta correta, o indivíduo recebe um ponto; a pontuação varia de 0 a 30 pontos e é avaliada da seguinte maneira: de 30 a 26 pontos, a função cognitiva está íntegra; entre 25 e 24 pontos, o paciente possui pequena alteração sugestiva de déficit; e com 23 pontos ou menos, o teste sugere possível déficit de cognição. ${ }^{14,15}$

O formulário desenvolvido pelas pesquisadoras inclui informações como dados sociodemográficos (nome, idade, escolaridade, estado civil, profissão 
e renda familiar), dados relativos ao perfil saúdedoença (IMC, atividade física, tabagismo, atividade sexual, acompanhamento ginecológico, uso de medicamentos, gestações e partos, presença de doenças crônicas, infecções urinárias, constipação intestinal e reposição hormonal) e sobre os sinais e sintomas (perda de urina, frequência, quantidade, situação e início da perda, presença de polaciúria, dor na bexiga, noctúria, perda durante o ato sexual e uso de métodos de adaptação).

Para avaliação da autoestima, empregaram-se questões do questionário de autoimagem e autoestima desenvolvido por Steglich ${ }^{16}$ que apresenta 78 itens a respeito desses dois domínios. Para aplicação nesta pesquisa, foram utilizadas as questões relacionadas somente à autoestima $(3,5$, $7,8,10,12,14,15,16,23,24,28,29,30,31,33$, $34,35,39,40,41,42,43,44,45,46,47,48,49$, $50,52,53,54,60,64,66,67,68,76,77,78)$. Cada questão apresenta cinco alternativas de respostas (cada resposta apresenta uma determinada pontuação, variando de 1 a 5 , sendo que ao final obtém-se um escore somatório de todos os itens estudados). Quanto maior a pontuação, melhores são a autoimagem e a autoestima. Para a avaliação, utilizaram-se pontos de corte para classificar a autoestima em baixa ou alta, utilizando a pontuação sugerida por Mazo, Cardoso \& Aguiar ${ }^{17}$ : autoestima - baixa: valores entre $41 \mathrm{e}$ 163; alta: valores 164 e 205. Esse instrumento apresenta alto índice de validade e fidedignidade (coeficiente de fidedignidade de Pearson 0,89). ${ }^{16}$

Os dados foram tabulados no software Excel e analisados no programa estatístico SPSS. O tratamento estatístico foi descritivo, tendo sido utilizado o teste de Kolmogorov-Smirnov para verificar a normalidade dos dados e testes nãoparamétricos (Qui-quadrado) com nível de significância de $\mathrm{p}<0,05$.

\section{RESULTADOS}

A idade média das idosas participantes foi de $67,59$ ( $\pm 5,16)$, variando de 60 a 80 anos; em relação à escolaridade, 12 (44,4\%) possuem ensino médio ou curso superior; $20(74,1 \%)$ não possuem vida conjugal; $11(40,7 \%)$ possuem renda familiar de um a dois salários mínimos (tabela 1$)$.

Tabela 1- Descrição geral da amostra em número e percentual em relação às variáveis sociodemográficas. Goiânia, GO, 2009.

\begin{tabular}{|c|c|c|}
\hline \multirow{2}{*}{ Variáveis } & \multicolumn{2}{|c|}{ Número de indivíduos } \\
\hline & $\mathrm{n}$ & $\%$ \\
\hline \multicolumn{3}{|l|}{ Faixa etária } \\
\hline Entre 60 a 69 anos & 20 & 74,1 \\
\hline Entre 70 a 79 anos & 6 & 22,2 \\
\hline 80 anos ou mais & 1 & 3,7 \\
\hline \multicolumn{3}{|l|}{ Escolaridade } \\
\hline Sabe ler e escrever ou primário incompleto & 1 & 3,7 \\
\hline Primário completo & 7 & 25,9 \\
\hline Ginásio & 7 & 25,9 \\
\hline Colegial ou curso superior & 12 & 44,4 \\
\hline \multicolumn{3}{|l|}{ Estado Civil } \\
\hline Com vida conjugal (casado, amasiado) & 7 & 25,9 \\
\hline Sem vida conjugal (solteiro, viúvo, divorciado) & 20 & 74,1 \\
\hline \multicolumn{3}{|l|}{ Renda Familiar } \\
\hline 1 a 2 salários mínimos & 11 & 40,7 \\
\hline 3 a 4 salários mínimos & 10 & 37,0 \\
\hline 5 a 8 salários mínimos & 4 & 14,8 \\
\hline Mais de 8 salários mínimos & 2 & 7,4 \\
\hline Total & 27 & 100,0 \\
\hline
\end{tabular}


A respeito do estado cognitivo avaliado por meio do MEEM, nenhuma das idosas apresentou alteração.

Quanto ao perfil de saúde-doença, nove idosas $(33,3 \%)$ apresentam sobrepeso e sete $(25,9 \%)$, obesidade; $19(70,3 \%)$ praticam atividade física. Das idosas estudadas, 21 $(77,8 \%)$ não mantêm atividade sexual; 22
(81,5\%) fazem acompanhamento ginecológico; $23(85,1 \%)$ utilizam algum tipo de medicamento; $18(66,7 \%)$ tiveram até três partos, 16 (59,3\%) tiveram parto vaginal, quatro $(14,8 \%)$ tiveram parto cesareana e três $(11,1 \%)$, ambos. Nenhuma idosa relatou ter infecções urinárias; seis $(22,2 \%)$ relataram possuir constipação intestinal e sete $(25,9 \%)$ fazem reposição hormonal (tabela 2 ).

Tabela 2 - Descrição geral da amostra em número e percentual em relação às variáveis saúde-doença. Goiânia, GO, 2009.

\begin{tabular}{|c|c|c|}
\hline \multirow{2}{*}{ Variáveis } & \multicolumn{2}{|c|}{ Número de indivíduos } \\
\hline & $\mathrm{n}$ & $\%$ \\
\hline \multicolumn{3}{|l|}{ IMC } \\
\hline Menor que 20 (abaixo do peso) & 2 & 7,4 \\
\hline 20 a 25 (normal) & 9 & 33,3 \\
\hline De 25,1 a 29,9 (sobrepeso) & 9 & 33,3 \\
\hline De 30,0 a 39,9 (obeso) & 7 & 25,9 \\
\hline \multicolumn{3}{|l|}{ Atividade física } \\
\hline Não & 8 & 29,6 \\
\hline Duas vezes na semana & 6 & 22,2 \\
\hline Três vezes ou mais & 13 & 48,1 \\
\hline \multicolumn{3}{|l|}{ Atividade sexual } \\
\hline $\operatorname{Sim}$ & 6 & 22,2 \\
\hline Não & 21 & 77,8 \\
\hline \multicolumn{3}{|l|}{ Acompanhamento ginecológico } \\
\hline $\operatorname{Sim}$ & 22 & 81,5 \\
\hline Não & 5 & 18,5 \\
\hline \multicolumn{3}{|l|}{ Medicamentos } \\
\hline Não & 4 & 14,8 \\
\hline Entre 1 e 3 & 11 & 40,7 \\
\hline 3 ou mais & 12 & 44,4 \\
\hline \multicolumn{3}{|l|}{ Número de partos } \\
\hline Até 3 & 18 & 66,7 \\
\hline Entre 4 e 7 & 7 & 25,9 \\
\hline 8 ou mais & 2 & 7,4 \\
\hline \multicolumn{3}{|l|}{ Infecção urinária } \\
\hline $\operatorname{Sim}$ & 0 & 0,0 \\
\hline Não & 27 & 100,0 \\
\hline \multicolumn{3}{|l|}{ Constipação intestinal } \\
\hline $\operatorname{Sim}$ & 6 & 22,2 \\
\hline Não & 21 & 77,8 \\
\hline \multicolumn{3}{|l|}{ Reposição hormonal } \\
\hline $\operatorname{Sim}$ & 7 & 25,9 \\
\hline Não & 20 & 74,1 \\
\hline Total & 27 & 100,0 \\
\hline
\end{tabular}


Em relação aos sinais e sintomas de IU, 12 $(44,4 \%)$ idosas apresentam perda de urina; destas, sete $(58,33 \%)$ apresentam perda em média uma vez na semana. Todas as idosas relataram perder pouca quantidade de urina e sete $(58,4 \%)$ relataram perda de urina aos esforços e após uma sensação de urgência. Quanto ao início dos sintomas, oito $(66,7 \%)$ apresentam perda há mais de um ano. $\mathrm{O}$ aumento da frequência miccional foi relatado por 12 idosas $(44,4 \%)$; três $(11,1 \%)$ possuem dor na bexiga e 17 (63\%) apresentam noctúria. Apenas uma idosa relatou ter perda de urina no ato sexual e quatro $(33,3 \%)$ recorrem a algum método de adaptação (tabela 3 ).

Tabela 3 - Descrição geral da amostra $(n=27)$ em relação às variáveis sinais e sintomas de IU e autoestima. Goiânia, GO, 2009.

\begin{tabular}{|c|c|c|c|c|}
\hline \multirow[t]{2}{*}{ Variáveis - sinais e sintomas IU } & \multicolumn{2}{|c|}{$\begin{array}{l}\text { Número de idosas de } \\
\text { acordo com a } \\
\text { classificação da auto } \\
\text { estima }\end{array}$} & \multirow[b]{2}{*}{ Total } & \multirow[t]{2}{*}{$\mathrm{p}^{*}$} \\
\hline & Alta & Baixa & & \\
\hline \multicolumn{5}{|l|}{ Perda de urina } \\
\hline Sim & 3 & 9 & 12 & \multirow{3}{*}{0,756} \\
\hline Não & 3 & 12 & 15 & \\
\hline Total & 6 & 21 & 27 & \\
\hline \multicolumn{5}{|l|}{ Frequência de perda de urina } \\
\hline Várias vezes ao dia & 0 & 5 & 5 & \multirow{3}{*}{0,901} \\
\hline Uma vez na semana & 3 & 4 & 7 & \\
\hline Total & 3 & 9 & 12 & \\
\hline \multicolumn{5}{|l|}{ Quantidade de perda de urina } \\
\hline Pouca & 3 & 9 & 12 & \multirow{3}{*}{-} \\
\hline Muita & 0 & 0 & 0 & \\
\hline Tot al & 3 & 9 & 12 & \\
\hline \multicolumn{5}{|l|}{ Início das perdas de urina } \\
\hline Há menos de um ano & 1 & 3 & 4 & \multirow{3}{*}{1,000} \\
\hline Há mais de um ano & 2 & 6 & 8 & \\
\hline Total & 3 & 9 & 12 & \\
\hline \multicolumn{5}{|l|}{ Polaciúria } \\
\hline Sim & 2 & 10 & 12 & \multirow{3}{*}{0,535} \\
\hline Não & 4 & 11 & 15 & \\
\hline Total & 6 & 21 & 27 & \\
\hline \multicolumn{5}{|l|}{ Dor na bexiga } \\
\hline $\operatorname{Sim}$ & 1 & 2 & 3 & \multirow{3}{*}{0,623} \\
\hline Não & 5 & 19 & 24 & \\
\hline Total & 6 & 21 & 27 & \\
\hline \multicolumn{5}{|l|}{ Situação de perda } \\
\hline Tosse, espirro, riso, ao subir escadas, ao andar & 0 & 2 & 2 & \multirow{4}{*}{0,144} \\
\hline Seguido de uma sensação de urgência para urinar & 2 & 1 & 3 & \\
\hline Nas duas situações anteriores & 1 & 6 & 7 & \\
\hline Total & 3 & 9 & 12 & \\
\hline \multicolumn{5}{|l|}{ Noctúria } \\
\hline Sim & 4 & 13 & 17 & \multirow{3}{*}{0,831} \\
\hline Não & 2 & 8 & 10 & \\
\hline Total & 6 & 21 & 27 & \\
\hline \multicolumn{5}{|l|}{ Perda no ato sexual } \\
\hline Sim & 1 & 0 & 1 & \multirow{3}{*}{0,057} \\
\hline Não & 5 & 21 & 26 & \\
\hline Total & 6 & 21 & 27 & \\
\hline \multicolumn{5}{|l|}{ Métodos de adaptações } \\
\hline $\operatorname{Sim}$ & 0 & 4 & 4 & \multirow{3}{*}{0,157} \\
\hline Não & 3 & 5 & 8 & \\
\hline Total & 3 & 9 & 12 & \\
\hline
\end{tabular}

*Qui-quadrado 
Das mulheres avaliadas $21(77,8 \%)$ possuem baixa autoestima (figura 01). Ao relacionar a autoestima aos sinais e sintomas de IU, verificou-se que não houve diferença estatisticamente significativa $(\mathrm{p}<0,05)$ entre as variáveis (tabela 3 ).

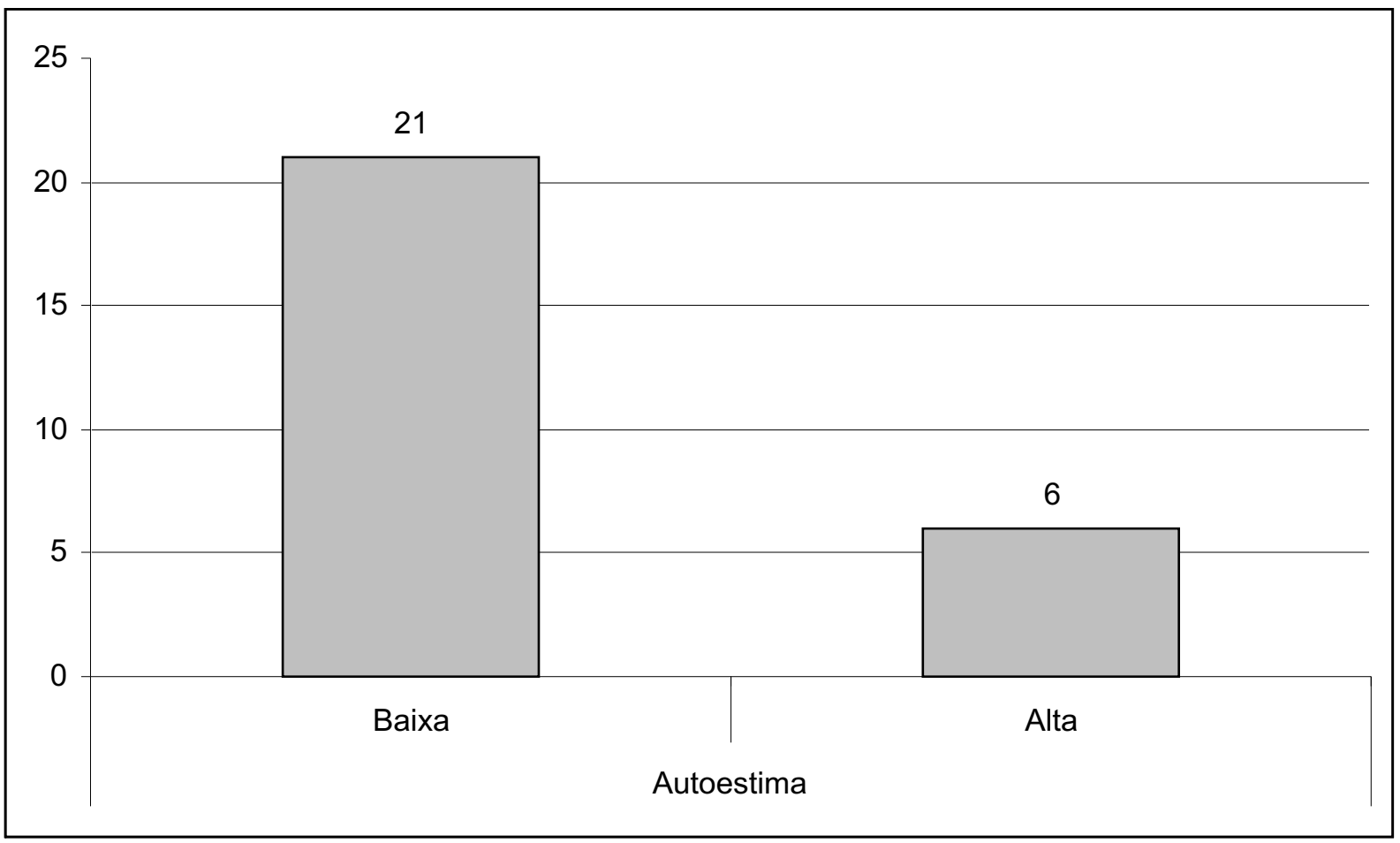

Figura 1 - Descrição da amostra (n = 27) em relação à variável autoestima. Goiânia, GO, 2009.

\section{DISCUSSÃO}

Os indivíduos participantes deste estudo são do sexo feminino, com idade entre 60 e 80 anos, sendo que das 27 participantes, 44,4\% apresentaram perda de urina. Ko et al., ${ }^{18} \mathrm{em}$ seu estudo com 141.815 indivíduos de ambos os sexos, verificaram prevalência de incontinência urinária em $22.054(64,3 \%)$ mulheres.

Grodstein et al. apud Oliveira et al. ${ }^{19}$ observaram que a prevalência da perda urinária aumenta com o avanço da idade, sendo que mulheres entre $61 \mathrm{e}$ 65 anos tiveram risco aumentado em $22 \%$, enquanto que naquelas com mais de 70 anos o risco foi de $77 \%$, quando comparadas a mulheres com idade inferior a 50 anos. Já em outro estudo realizado por Pastor et al..$^{20} \mathrm{com} 1.151$ indivíduos, sendo 567 mulheres, não se observou aumento da prevalência da incontinência urinária entre as mulheres com o avanço da idade.
No presente estudo, em relação à escolaridade, $15(55,5 \%)$ possuem até ensino fundamental; 20 (74,1\%) não possuem vida conjugal; 11 (40,7\%) possuem renda familiar entre um e dois salários mínimos. Dados semelhantes foram observados por Guarisi et al. ${ }^{21}$ em estudo com mulheres climatéricas, cuja maioria era branca, com baixo grau de escolaridade e não possuía emprego remunerado; a maioria, no entanto, vivia com companheiro.

Higa, Lopes \& Reis, ${ }^{4}$ em revisão de literatura, afirmam que a obesidade é um fator que agrava ou contribui para a incontinência urinária, juntamente com outros fatores como paridade, tipo de parto, mudanças hormonais durante a menopausa, constipação intestinal, presença de doenças crônicas, uso de determinados medicamentos e tabagismo. No presente estudo, foi observado que $59,2 \%$ das idosas apresentaram sobrepeso ou obesidade; $85,1 \%$ utilizavam algum 
tipo de medicamento; $66,7 \%$ tiveram até três partos e 59,3\%, parto vaginal.

De acordo com os tipos de IU, a maioria $(58,4 \%)$ referiu sintomas de IUM, seguido de $25 \%$ com sintomas de IUU e $16,6 \%$ de IUE, corroborando o estudo de Silva \& Lopes. ${ }^{22}$ Lopes $\&$ Higa, ${ }^{5}$ em estudo com mulheres com queixa de IU, verificaram que a queixa de IUE ocorre em média entre mulheres mais jovens, enquanto a IUM e IUU predominaram nas mulheres com média de idade maior.

No estudo aqui apresentado, 58,33\% apresentaram perda em média uma vez por semana e todas as idosas relataram perder pouca quantidade de urina. No estudo de Silva $\&$ Lopes, ${ }^{22}$ observou-se que a maioria (60\%) acreditava perder uma pequena quantidade de urina; no entanto, $57,1 \%$ das mulheres referiram perda de urina diversas vezes ao dia e apenas $20 \%$ relataram perda uma vez por semana ou menos.

Da amostra analisada, $66,7 \%$ apresentaram perda de urina há mais de um ano e 33,3\% recorriam a algum método de adaptação. Tamanini et al., ${ }^{23}$ em estudo observacional com 156 mulheres com queixa de IU, encontraram que $94,2 \%$ declararam perder urina há mais de um ano e $61 \%$ usavam absorventes como estratégia para minimizar os efeitos da IU.

Dentre as participantes deste estudo, $81,5 \%$ declararam fazer acompanhamento ginecológico. Por outro lado, Guarisi et al., ${ }^{24}$ em estudo do tipo inquérito populacional domiciliar com mulheres com sintomatologia do climatério, avaliaram a procura por cuidados médicos devido à queixa de IU, encontrando que pouco mais da metade $(58,8 \%)$ consultou um médico por este motivo. Dentre os motivos relatados pela não procura, os mais mencionados foram que os sintomas não mereciam atenção médica, não eram tão fortes ou não incomodavam.

Os resultados obtidos pelo questionário de autoestima revelaram que $77,8 \%$ das participantes apresentaram baixa autoestima. Entretanto, não se verificou relação entre a autoestima e os sinais e sintomas de IU. Da mesma forma, no estudo realizado por Ferolla ${ }^{13}$ com mulheres com diagnóstico de IUE, não houve relação entre a autoestima e as características clínicas da incontinência. Porém, no estudo citado, foi possível verificar correlação entre a IU e as características sociodemográficas, sendo que os baixos valores de autoestima estavam ligados a pouca escolaridade, renda familiar baixa e faixa etária inferior a 40 anos.

Hajjar ${ }^{25}$ sugere que a IU confere aos indivíduos impacto psicológico e social, trazendo maior nível de constrangimento, ansiedade, isolamento social e depressão, podendo ser influenciado por fatores culturais, sociais e psicológicos. Afirmam que as alterações psicossociais variam de um indivíduo para outro, conforme a faixa etária e as particularidades da IU. Menor grau de comprometimento foi observado em mulheres idosas e que apresentavam pequena quantidade de perda de urina.

Higa, Lopes \& Turato $^{26}$ afirmam que as mulheres com mais de 60 anos associam a IU à idade avançada e ao número de partos, sendo uma condição inerente a sua história de vida. Essas mulheres consideraram tal condição uma ameaça para sua autoestima, o que as incentivava desenvolver sistemas adaptativos, e caso estes fossem bem-sucedidos, poderiam aceitar a IU e conduzir uma vida "normal".

Lopes \& $\mathrm{Higa}^{5}$ investigaram as restrições causadas pela IU na vida da mulher, que envolveram a vida sexual, sobretudo por perder urina e sentir dor durante a relação; a vida social, por impedi-la de sair de casa; e as atividades diárias e profissionais, por interromperem os mesmos constantemente para usar o banheiro.

Miner ${ }^{27}$ afirma que a autoestima diminuída está relacionada à falta de controle sobre seu corpo, preocupação com a higiene, problemas psicológicos e afastamento da família e dos amigos. Além disso, podem ocorrer isolamento social, depressão, tristeza, ansiedade e impacto na qualidade de vida.

Alguns autores avaliaram a qualidade de vida em mulheres comIU. Martínez et al. ${ }^{28}$ avaliaram mulheres com IUE e observaram pior qualidade de vida em 
relação ao impacto da IU e limitação na atividade física. Oliveira et al. ${ }^{7}$ correlacionaram a qualidade de vida aos sintomas urinários, percebendo que a relação entre os sintomas e suas influências não apresentou evidências de agravos intensos na vida das mulheres, com exceção do sono e disposição com enurese e piora emocional com urgência.

Dados semelhantes foram observados por Auge et al., ${ }^{11}$ que avaliaram a qualidade de vida em mulheres submetidas ou não ao tratamento cirúrgico. $\mathrm{O}$ grupo de mulheres que não realizou tratamento para IU apresentou diminuição da disposição física e qualidade do sono, prejuízos na realização de atividades físicas, em casa e no trabalho. Os resultados apontaram ainda impacto negativo nas relações familiares, amizades, parceiro e nas relações sexuais.

\section{CONCLUSÃO}

Observou-se, neste estudo, que $44,4 \%$ das idosas relataram perder urina, a maioria

\section{REFERÊNCIAS}

1. Abrams, et al.. Significado de ter incontinência urinaria e ser incontinente na visão das mulheres. Texto contexto - enferm. 2008; 17(3): 527-35.

2. Abrams et ,al. Restrições causadas pela incontinência urinaria à vida da mulher. Rev. esc. enferm. USP. 2006; 40 (1): 34-41.

3. Robles JE. La Incontinencia urinaria:anales del sistema sanitario de navarra. 2006; 29 (2): 219-31.

4. Higa R, Lopes MHBM, Reis MJ. Fatores de risco para incontinência urinária na mulher. Rev. esc. enferm. USP. 2008; 42 (1): 187-92.

5. Lopes MHBM, Higa R. Restrições causadas pela incontinência urinaria à vida da mulher. Rev. esc. enferm 2006; 40 (1): 34-41.

6. Honório MO, Santos SMA. Incontinência urinária e envelhecimento: impacto no cotidiano e na qualidade de vida. Rev Bras Enferm 2009; 62 (1): 51-6.

7. Oliveira JMS, et al. Correlação entre sintomas urinários e qualidade de vida em mulheres com incontinência urinária. Fisioter Pesq 2007; 14 (3); 12-7. perdendo pouca quantidade, em média uma vez por semana, tendo os sintomas se iniciado há mais de um ano.

Apesar de apresentarem baixa autoestima, esta não estava correlacionada aos sinais e sintomas da IU. A baixa autoestima é uma condição não rara em idosos, sendo que vários fatores podem contribuir, além da IU. O impacto da incontinência na vida das mulheres sofre alterações conforme a idade, renda familiar, grau de escolaridade, tipo de incontinência, frequência e quantidade das perdas, além dos fatores culturais e sua percepção de saúde.

O número reduzido e as características da amostra avaliada podem ter influenciado os resultados deste estudo, constituindo, assim, limitação para o mesmo, sendo que os resultados encontrados também não devem ser generalizados. Faz-se necessário realizar novas pesquisas com a população de mulheres idosas, para que se conheçam seus sentimentos em relação à IU, condição frequente nesta faixa etária.
8. Reis $\mathrm{RB}$, et al. Incontinência urinária no idoso. Acta Cir. Bras. 2003; 18 (5): 47-51.

9. Rett MT, et al. Qualidade de vida em mulheres após tratamento da incontinência urinária de esforço com fisioterapia. Rev. Bras. Ginecol. Obstet 2007; 29 (3): 134-40.

10. Borba AMC, Lelis MAS, Bretãs ACP. Significado de ter incontinência urinaria e ser incontinente na visão das mulheres. Text context enferm. 2008; 17 (3): 527-35.

11. Auge AP, et al. Comparações entre os índices de qualidade de vida em mulheres com incontinência urinária submetidas ou não ao tratamento cirúrgico. Rev. Bras. Ginecol. Obstet. 2006; 28 (6): 352-57.

12. Abreu NS, et al. Qualidade de vida na perspectiva de idosas com incontinência urinária. Rev. Bras. Fisioter. 2007; 11(6): 429-36.

13. Ferolla EC. A auto-estima de mulheres portadoras de incontinência urinária de esforço. 2001. Dissertação (Mestrado em Enfermagem) Universidade de São Paulo, São Paulo ; 2001.105p 
14. Nagato AC, et al. Avaliação cognitiva de idosas institucionalizadas através do Mini Exame do Estado Mental com ou sem tratamento fisioterapêutico. Fisioter Brasil. 2007; 8 (4): 233-38.

15. Oliani MM, et al. Locomoção e desempenho cognitivo em idosos institucionalizados com demência. Fisioter. Mov. 2007; 20 (1); 109-14.

16. Steglich LA. Terceira Idade, aposentadoria, autoImagem e auto-estima. Dissertação.[ Mestrado em Educação]_Universidade Federal do Rio Grande do Sul; 1978.

17. Mazo GZ, Cardoso FL, Aguiar DL. Programa de hidroginástica para idosos: motivação, autoestima e auto-imagem. Rev Bras Cineantropom Desempenho Hum 2006; 8 (2): 67-72.

18. Ko Yu, et al. The impact of urinary incontinence on quality of life of the elderly. Am J Mang Care 2005; 11 (4): 103-11.

19. Grodstein et al. Correlação entre sintomas urinários e qualidade de vida em mulheres com incontinência urinária. Fisioter Pesq. 2007; 14 (3): 12-7.

20. Pastor MV, et al. Prevalencia de la incontinencia urinaria y factores asociados en varones y mujeres de más de 65 años. Aten Primaria. 2003; 32 (6); 337-42.
21. Guarisi T, et al. Incontinência urinária entre mulheres climatéricas brasileiras: inquérito domiciliar. Rev. Saúde Pública. 2001; 35 (5): 228-35.

22. Silva L, Lopes MHBM. Incontinência urinária em mulheres: razões da não procura por tratamento. Rev. esc. enferm 2009; 43 (1): 72-8.

23. Tamanini JTN, et al. Validação do "King's Health Questionnaire” para o português em mulheres com incontinência urinária. Rev. Saúde Pública. 2003; 37 (2): 203-11.

24. Guarisi T, et al. Procura de serviço médico por mulheres com incontinência urinária. Rev. Bras. Ginecol. Obstet. 2001; 23 (7): 439-43.

25. Hajjar RR. Psychosocial impact of urinary incontinence in the elderly population. Clin. Geriatr. Med. 2004; 20: 553-64.

26. Higa R, Lopes MHBM, Turato ER. Significados psicoculturais da incontinência urinária feminina: uma revisão. Rev Lat Am Enfermagem. 2008; 16 (4): 779-86.

27. Miner Jr PB. Economic and personal impact of fecal and Urinary Incontinence. Gastroenterol 2004; 126 (1): 8-13.

28. Martínez $\mathrm{CB}$, et al. Calidad de vida en las pacientes con incontinencia urinaria. Actas Urol Esp. 2008; 32 (2): 202-10. 